\title{
La enseñanza de los gestos emblemáticos en la competencia léxico-semántica: una propuesta para el aula de ELE
}

\author{
HELENA SOFÍA BELÍO-APAOLAZA \\ Massachusetts Institute of Technology \\ hbelio@mit.edu
}

\section{Resumen}

En este trabajo se aboga por la incorporación de los gestos emblemáticos en los contenidos que deben enseñarse en la clase de ELE. A partir de las características lingüísticas de estos gestos y el léxico, así como en la similitud de los procesos cognitivos que aparecen en el aprendizaje de ambos, se defiende la integración de los emblemas dentro del desarrollo de la competencia léxica. Para ello, se ofrecen una serie de criterios de graduación en los diferentes niveles de lengua y de integración en el aula que faciliten la tarea docente a la hora de su incorporación en los programas y materiales didácticos. Además, se ahonda en algunas consideraciones que deben tenerse en cuenta a la hora de trabajar estos gestos interculturalmente, es decir, comparando el código gestual materno y el del español, e intraculturalmente, esto es, reconociendo diferencias dentro del mundo panhispánico.

\section{Palabras clave}

Emblemas; gestos emblemáticos; comunicación no verbal; competencia léxica; español lengua extranjera; ELE. 


\title{
Teaching emblematic gestures within Lexical Competence: a proposal for the Spanish Classroom
}

\begin{abstract}
This paper advocates the incorporation of emblematic gestures as part of the content that must be taught in the SFL classroom. Given the lack of theoretical and pedagogical research on the teaching of emblems and as a result of the linguistic characteristics of these gestures and the lexicon as well as the similarity of the cognitive processes that appear in the study of both, we defend the integration of emblems within the development of Lexical Competence. To this end, in order to facilitate the incorporation of these gestures in Spanish programs and materials, we present a series of graduated criteria as well as criteria for integration in the different language levels in the classroom. In addition, we explain some considerations that must be taken into account when working with these gestures interculturally, that is, comparing the maternal and Spanish gestural code, and intraculturally, in other words, recognizing differences within the PanHispanic world.
\end{abstract}

\section{Keywords}

Emblems; emblematic gestures; Lexical Competence; Spanish as a Foreign Language.

Recibido el 04/11/2019

Aceptado el 15/01/2020 


\section{Introducción}

La enseñanza de los signos no verbales en el aula de ELE resulta parte imprescindible para garantizar a nuestros estudiantes una comunicación intercultural exitosa. No obstante, como advierte Cestero (2017b: 344), todavía no se ha comenzado una incorporación sistemática en los programas de L2/LE. Según esta autora (2017b: 345), la ausencia generalizada de los elementos de comunicación no verbal en la mayoría de manuales se debe a la falta de atención explícita por parte del PCIC (Instituto Cervantes, 2006) a los signos no verbales. Para corroborar lo anterior, se realizó una búsqueda exhaustiva por dos de los principales enfoques curriculares europeos, el MCER (Consejo de Europa, 2002, 2018) y el PCIC (Instituto Cervantes), así como por las diversas publicaciones de ACTFL $(2012,2015)$ y NCSSFL-ACTFL $(2014,2017)$, ambas reconocidas instituciones sobre la enseñanza de lenguas en el ámbito estadounidense. En esta búsqueda, se puede dar cuenta de la poca sistematización y dedicación directa que reciben la $\mathrm{CNV}$, puesto que en ninguno de los documentos consultados aparecen pautas para la integración de los signos no verbales en el aula, ni se explica detalladamente cómo incluirlos a lo largo de los diferentes niveles de lengua (Belío-Apaolaza, 2018: 145-179).

En este trabajo se trata de contribuir a cubrir ese vacío curricular ofreciendo una serie de criterios para facilitar la incorporación inmediata de los gestos emblemáticos junto con el aprendizaje del léxico en el aula de ELE. Estos gestos pueden definirse sucintamente como signos kinésicos que poseen una correspondencia léxica directa en la lengua verbal y cuya interpretación no es ambigua dentro de la comunidad de habla que comparte la asociación significado-significante correspondiente. Por ejemplo, el gesto que se realiza girando el dedo índice alrededor de la sien, cuyo significado es 'estar loco', es un emblema. Para el campo en el que se enmarca este trabajo, la enseñanza de ELE, es importante destacar la variabilidad cultural de estos gestos. Poyatos (1994: 187), a este respecto, los define como «un gesto que tiene un equivalente verbal sin ninguna ambigüedad en su propia cultura». Dentro de los emblemas existe una gran variación no solo intercultural, sino también intracultural, esto es, entre miembros que comparten una misma lengua e, incluso, cultura. Es por ello que deberán ser incluidos en el aula, no solo para que los estudiantes aprendan las diferencias 
emblemáticas entre su lengua materna y el español, sino también para que conozcan las diferencias intrahispánicas.

Por ejemplo, del mismo modo que un estudiante japonés de español deberá aprender que para お金 (okane) su correspondencia léxica en español es dinero, también podrá aprender que para el gesto que tiene ese significado en japonés, el cual se realiza situando la palma de la mano hacia arriba y formando un círculo con los dedos índice y pulgar mientras que el resto de dedos estirados y separados apuntan hacia el lado contrario de la mano que se utiliza ${ }^{1}$, en español se realiza con las yemas de los dedos índice y corazón de una mano, con sus puntas situadas hacia arriba, rozando con un movimiento rápido y repetido la yema del pulgar ${ }^{2}$. Además, si los alumnos están estudiando el español de las variedades de México u Honduras, deberán conocer que dentro del repertorio emblemático de ambos países existe otro gesto que también significa 'dinero', el cual se realiza con «el pulgar y el índice de la mano derecha (resto del puño cerrado) en forma de herradura paralelamente al piso o también sesgados, con las yemas separadas a unos 4-5 cm., y representándose así una $C$ con la abertura hacia la izquierda» ${ }^{3}$ (Meo-Zilio y Mejía, 1980: 121). Asimismo, deberán saber que, además de la palabra dinero, a este emblema también pueden asociarse otras unidades léxicas pertenecientes al registro coloquial, por ejemplo, baro o lana en México.

Los ejemplos anteriores muestran la productividad de que la competencia emblemática, esto es, el conocimiento y la capacidad de usar los emblemas en una lengua, se desarrolle en el aula y se estudie por parte de los investigadores junto con la competencia léxica, en lo cual profundizamos a continuación. A la hora de referirnos a ejemplos concretos, se seguirá la siguiente convención: la denominación del emblema se escribirá entre barras oblicuas, es decir /denominación/; el significado estará delimitado por comillas simples, esto es, 'significado del emblema'; y las unidades léxicas asociadas estarán marcadas en letra cursiva, o sea, unidades léxicas asociadas al

\footnotetext{
1 Este gesto puede visualizarse en el siguiente vídeo: http://bit.ly/2Ziz7uE (El Vicho, 2018, minuto 1:08).

${ }^{2} \mathrm{Su}$ realización puede observarse en el vídeo a continuación: http://bit.ly/2MbU6gB (Velo PuigDurán y Gómez Sancha, 2017, minuto 2:49).

${ }^{3} \mathrm{Su}$ realización puede observarse en el minuto 11:10 del episodio «Todos somos Cuervos» (nº 12, temporada 4) de la serie de Netflix Club de Cuervos (Chiver, Vargas y Alazraki, 2019).
} 
emblema. La descripción de la realización (las partes del cuerpo que participan y su lugar articulatorio, la dirección, la orientación o la velocidad del movimiento) se presentarán en letra redonda. En todos los casos donde sea posible, se incluirá una nota con una referencia audiovisual para que el lector pueda observar su realización kinésica y su uso en la lengua oral.

\section{La relación entre los emblemas y el léxico}

\subsection{Estudios teóricos y neurolingüísticos}

La relación entre los gestos emblemáticos y el léxico ha sido abordada por varios autores. Dahan y Cosnier (1977), de hecho, se refieren a ellos como gestos cuasi-lingüísticos. Kendon (1988: 136), por su parte, describe el proceso de lexicalización de los gestos de la siguiente manera: «gestures become fully lexicalized when, for one reason or another, speech cannot be used for prolonged periods, but when, nevertheless, all of the functions of spoken interchange are required». Payratò (2003: 76) considera que los emblemas son una categoría gestual similar a la categoría verbal de las interjecciones, ya que ambos «[are] designed to encapsulate different messages in a very autonomous way». Para Matsumoto y Hwang (2013: 2), estos gestos son mensajes verbales codificados en movimientos corporales, producidos espontáneamente y entendidos como una forma de comunicación.

Desde un punto de vista neurolingüístico, algunos estudios han demostrado que el control del lenguaje verbal y el de los gestos comparten el mismo sistema neuronal (Gentilucci, Dalla Volta y Gianelli, 2008). Específicamente, hay investigadores que sugieren que los sistemas de control situados en el córtex frontal del hemisferio izquierdo, donde se sitúa el área de Broca, combinan la información de los emblemas y del lenguaje verbal (Willems, Ózyürek y Hagoort, 2007) y que la comprensión del significado de las unidades léxicas y de estos gestos se produce por parte de un sistema común y superpuesto de regiones del cerebro (Xu et al., 2009), lo cual sugiere que tanto el componente verbal como el gestual comparten los mismos mecanismos neuronales. Estos estudios apoyan la idea de que las palabras y los gestos emblemáticos forma un sistema de comunicación único (Barbieri et al., 2009).

Respecto a las propiedades semánticas y pragmáticas compartidas por los emblemas y los elementos verbales de la lengua, Poggi y Zomparelli (1987) señalan que 
las palabras y los gestos léxicos (categoría que definen como gestos que funcionan como componentes de un acto comunicativo cuyo acto performativo puede ser diferente en una situación u otra) comparten muchas propiedades, ya que sus significados se aprenden de una manera parecida y ambos participan en los mismos procesos semióticos. Dentro de esta categoría pueden incluirse los emblemas, ya que estos pueden desarrollar diferentes funciones comunicativas, por ejemplo, el emblema /comer $/ 4$ puede emplearse para preguntar si alguien quiere comer, para indicar que se quiere comer, para explicar que alguien ha ido a comer, etc. Estos autores sostienen que los gestos léxicos deben entenderse como participantes de los procesos lingüísticos en los que intervienen las unidades de la lengua oral, en lugar de clasificarse como parte de la comunicación no verbal o el paralenguaje.

Si bien consideramos que los emblemas deben concebirse como parte de la comunicación no verbal, puesto que son gestos que comunican de manera no verbal, apoyamos la idea de Poggi y Zomparelli (1987) que sostiene que los mecanismos que intervienen en la comunicación y los procesos subyacentes al aprendizaje de estos gestos están intrínsecamente relacionados con los procesos y mecanismos que participan en el léxico, como explicamos seguidamente.

\subsection{La competencia léxica y la competencia emblemática}

Para Lahuerta y Pujol (1996: 121, apud Gómez Molina, 2004: 484), la competencia léxica es «la capacidad de relacionar formas y significados y utilizarlos adecuadamente (...) y al lugar donde reside el conocimiento de esa competencia se le denomina lexicón mental». La definición anterior se puede perfectamente aplicar a los emblemas, puesto que estos gestos, como se explica en Belío Apaolaza (2018), están constituidos por cuatro elementos: forma (en otras palabras, su realización gestual), significado (esto es, la representación mental asociada a la forma/significante del emblema), uso (es decir, los rasgos pragmáticos y sociolingüísticos de su aparición en la comunicación) y exponentes lingüísticos asociados (o sea, las unidades léxicas o elementos verbales con

\footnotetext{
${ }^{4}$ Los dedos de una mano se apiñan, se acercan y se separan de la boca en movimientos rápidos y repetidos. Este gesto se encuentra en el siguiente vídeo: http://bit.ly/20pSQI1 (Garrido y Montalbán, 2018c, minutos 18:50 y 27:44). En la primera aparición, el gesto se realiza con ambas manos.
} 
los que puede darse el emblema en la comunicación), y la capacidad de relacionarlos adecuadamente determinará que un hablante sea competente en su uso lingüístico.

En relación con lo anterior, Marslen-Wilson (1989, apud Baralo, 1997: 62) sostiene que el lexicón se procesa a través de dos dominios: el representacional, donde se da «una relación con los datos sensoriales (análisis fonético acústico de la señal que entra en el habla) y el sistema de procesamiento léxico» y el computacional, donde se produce «una relación entre este sistema [el sistema de procesamiento léxico] y la representación de la información semántica y sintáctica asociada con la palabra que está siendo reconocida y analizada» (Baralo, 1997: 62). En los emblemas también se produce este procesamiento a través del dominio representacional, donde los datos sensoriales no son fonético-acústicos, sino que son gestuales, y mediante el dominio computacional, donde los aprendientes deben procesar el emblema junto con sus elementos semióticos y discursivos.

Es posible, por lo tanto, que en el lexicón mental no solo se almacenen las formas verbales y sus significados, sino que también se incluyan las formas gestuales, esto es, los emblemas, como parte de esa red mental donde se organizan las categorías que permiten denominar el mundo que nos rodea. A este respecto, es ilustrativo un ejemplo que esta autora experimentó como docente en sus cursos de español inicial en Estados Unidos. Varias clases después de haber aprendido el emblema /juntos/5, el cual se emplea para indicar que dos personas están saliendo o manteniendo una relación informal, una estudiante quiso expresar que una pareja había roto. Como no disponía de la palabra precisa para ello, en lugar de recuperar un léxico más simple dentro de ese campo semántico, por ejemplo, «no están juntos»o «no son novios ahora» (vocabulario que ya había estudiado), recurrió al gesto /juntos/, pero en lugar de dar golpes rápidos y cercanos en los cantos de los dedos índices de cada mano, realizó un solo golpe y después separó ambos dedos extendiendo los brazos hasta la anchura de su cadera o perfil, de manera que en lugar de representar que alguien se une, como sucede en /juntos/, quiso señalar que alguien se separaba. En otras palabras, realizó un proceso creativo a través de un elemento lingüístico que ya había interiorizado en su repertorio.

\footnotetext{
${ }^{5}$ Los dedos índices de ambas manos (con la palma apuntando al frente y el resto de dedos en puño cerrado) se golpean con la sección lateral externa en movimientos rápidos y repetidos con una separación aproximada de $5 \mathrm{~cm}$. Su realización puede observarse en el siguiente vídeo: http://bit.ly/2X8hInx (Subirats, 2018a, minuto 17:20).
} 
Esta construcción creativa es semejante a un tipo de transferencia negativa: el error de producción por creación de palabra nueva, también denominado transferencia creativa: «resulta del intento del estudiante de resolver la dificultad que supone no conocer un término de la LE, “creando” uno nuevo» (Kumaravadivelu, 1988, apud Durão, 2007: 40). En este caso, la estudiante no realizó una transferencia desde la lengua materna u otra lengua extranjera, sino que se trata de un proceso de transferencia emblemática a partir de la lengua meta.

Es decir, en su lexicón mental se habría integrado el emblema dentro de la red asociativa relacionada con las nociones de las relaciones sentimentales, de manera que cuando se estaba tratando un contenido relacionado con ese tema, recurrió a su conocimiento emblemático para suplir sus carencias léxicas y poder comunicarse. Esa transferencia creativa gestual podría asimilarse a lo que sucede en la creatividad léxica, sobre la cual Baralo (2001: 169) apunta que «es una estrategia común de los hablantes de LM y L2 ante un "problema léxico", esto es, ante una situación de uso en el que no disponen de la palabra o expresión adecuada en el léxico ya almacenado». En nuestro ejemplo, en lugar de recurrir a la creación de una palabra nueva para resolver un problema léxico, creó un nuevo gesto a partir del que ya había almacenado en su conocimiento lingüístico.

En este sentido, Marconi (1997) sostiene que la estructura de la competencia léxica se constituye por dos aspectos básicos que interaccionan: el referencial y el inferencial. En el primero, el mundo que nos rodea se proyecta a través del léxico. De esta manera, las palabras, como formas verbales, pueden equipararse a los emblemas, como formas no verbales con las que también expresamos ideas y conceptos. En el segundo, el inferencial, aparecen actuaciones (como el hallazgo de sinónimos, paráfrasis, definiciones, etc.) que permiten establecer una red de conexiones entre palabras. A partir de esta perspectiva, podemos establecer que en el emblema creado a partir de /juntos/, la estudiante llevó a cabo una tarea de referencia a través de un proceso inferencial semántico para designar un concepto dentro de la red paradigmática que había tejido en su lexicón mental, ya que, como sostiene Marconi (1997: 62), «(...) we use our inferential competence to carry out referential tasks when we lack direct referential competence».

Siguiendo con esta presencia de los emblemas en el lexicón mental, es interesante reflexionar sobre qué implica conocer una palabra. A partir de los trabajos de varios autores, Gómez Molina (2004) compendia una serie de condiciones a este respecto: 
(...) saber su denotación, tener suficiente información gramatical y funcional (...), reconocerla en su forma oral (pronunciación) y escrita (ortográfica), conocer cómo se relaciona con otras que podrían haber aparecido en su lugar (relaciones paradigmáticas), cómo se combina con otras (relaciones sintagmáticas), conocer sus registros y utilizarla de forma apropiada según la situación comunicativa, saber la información cultural que transmite y sus usos metafóricos, también si pertenece a alguna unidad fraseológica, recordarla y recurrir a ella cuando se necesite, etcétera. (Gómez Molina, 2004: 498)

De esta manera, podemos añadir que conocer una palabra o una unidad léxica también puede suponer conocer el emblema relacionado con ella y viceversa: conocer un emblema también será conocer las unidades léxicas asociadas a él. Es decir, dentro de un gesto emblemático se podrán establecer campos semánticos y relaciones paradigmáticas que permitan asociar los diferentes exponentes lingüísticos apropiados para su significado y uso. Por ejemplo, /blablablá/ ${ }^{6}$ podrá relacionarse con las unidades léxicas hablar, parlotear, cotillear, pero no con dialogar o enunciar. Por ello, será esencial llevar a cabo investigaciones que determinen los núcleos semánticos de los emblemas, ya que a partir de ellas se podrán establecer los campos semánticos y las redes paradigmáticas adecuadas que faciliten los estudios teóricos y las prácticas didácticas.

En relación con lo anterior, Gómez Molina (2004: 493), basándose en los principios de la lingüística cognitiva, subraya que la enseñanza del vocabulario debe integrarse dentro de secuencias donde se trabajen conjuntamente la estructura de las palabras, las relaciones paradigmáticas, las relaciones sintagmáticas y el valor discursivo. En el aprendizaje de los emblemas debe ocurrir lo mismo, deben integrarse todos sus elementos constitutivos: su estructura, esto es, su forma; su significado y su uso, donde también se tiene en cuenta el valor discursivo; y los exponentes lingüísticos asociados, es decir, donde se establecen las relaciones paradigmáticas en torno a un núcleo semántico.

\footnotetext{
${ }^{6}$ Los dedos de una mano se apiñan estirados y se separan en movimientos rápidos y repetidos de apertura y cierre completo de 5 a $8 \mathrm{~cm}$. hasta que las yemas del pulgar se tocan con las yemas del resto de los dedos. Su realización puede observarse en el siguiente vídeo: http://bit.ly/2VSbNBe (Nejokitablog, 2016, minuto 1:23).
} 
Siguiendo con las consideraciones del autor anterior, en el aprendizaje de las unidades léxicas se produce un proceso gradual que «pasa por diferentes estadios: comprensión o interpretación (por cualquiera de los dos procedimientos top-down o botton-up), utilización, retención (memoria a corto plazo) y fijación (memoria a largo plazo, reutilización)» (Gómez Molina, 2004: 498). Como se muestra en Belío Apaolaza (2018), en el aprendizaje de los gestos emblemáticos también se da un proceso similar: en primer lugar, el estudiante tiene que detectar la presencia de un gesto al que se le asocia un significado en la producción oral; en segundo lugar, debe comprenderlo e interpretarlo realizando una asociación entre la forma y el significado, así como llevando a cabo una contextualización discursiva de su uso; en tercer lugar, debe saber producirlo adecuadamente (teniendo en cuenta tanto la forma como el uso pragmático y sociolingüístico); y, en cuarto lugar, debe poder producirlo, tanto a corto como a largo plazo.

En este sentido, todo lo anterior nos conduce a formular una de las hipótesis que podrá guiar futuros estudios empíricos sobre el aprendizaje de estos gestos: cuando se aprende un nuevo emblema de una L2/LE, se llevan a cabo procesos similares a cuando se aprende una nueva palabra o unidad léxica. Basándonos en la terminología establecida en la taxonomía contrastiva de Belío Apaolaza (2018), podemos señalar que si se trata de un emblema dimorfo-sinónimo (es decir, un gesto en la L2/LE con el mismo significado que un gesto de la lengua materna pero con una forma diferente, por ejemplo, /buenísimo/ del español y /おいしい/ (/oishii/) (/delicioso/) del japonés ${ }^{7}$ ), a un mismo significado deberá dársele otra forma, como sucede, por ejemplo, cuando un anglohablante aprende la palabra mesa para hacer referencia a lo que él denomina table. Si se trata de un (cuasi)equimorfo-dimónimo (gesto con la misma forma o una similar en la L2/LE y en la lengua materna pero con significado diferente, por ejemplo, /delgado/del españoly /ガールフレンド/ (/gārufurendo/) (/novia/) del japonés ${ }^{8}$ ), se

\footnotetext{
${ }^{7}$ Ambos se utilizan para indicar que la comida está deliciosa, pero el español se realiza acercando y separando al labio las yemas de una mano apiñadas, con la opción de que se produzca un beso, mientras que el japonés se realiza deslizando el dorso de una mano por la barbilla, como apuntan Hamiru-aqui y Alieen Chang (2004: 44).

8 Estos gestos se realizan levantando el dedo meñique de una mano mientras que el resto de dedos permanecen cerrados en puño. Ambos pueden observarse en los siguientes vídeos: /delgado/ https://goo.gl/6wAbjy (RTVE, 2018, minuto 4:46) y http://bit.ly/2ECIgG0 (Subirats, 2018b, minuto
} 
tendrá que aprender que para la misma forma o una similar existe un significado diferente, como ocurre cuando un hispanohablante aprende las palabras sensible (forma verbal equivalente a un equimorfo) o constipated (equivalente a un cuasiequimorfo) en inglés o, al contrario, cuando un anglohablante aprende sensible o constipado. Si se trata de un (cuasi)equimorfo-sinónimo (por ejemplo, /dinero/ y /money/ en español e inglés, que se realizan en ambas lenguas frotando la yema del pulgar con la de los dedos índice y corazón), solo se tendrá que transferir el conocimiento gestual materno a la lengua extranjera, como sucede en pizza (parangonable a un equimorfo) o en list-lista (equivalente a un cuasiequimorfo) en inglés y español. Si se trata de un unimorfo (esto es, un emblema sin un equivalente ni en forma ni en significado), se deberá emblematizar un concepto que no posee una forma gestual específica en la lengua materna (por ejemplo, cuando un anglohablante aprende /delgado/), como sucede en la palabra del inglés toes para un hispanohablante. Es decir, algunos conceptos están lexicalizados de la misma manera que algunos conceptos están emblematizados en una lengua, pero no en otra.

Como se comprobó en la investigación de Belío Apaolaza (2018), en ese proceso de aprendizaje, como ocurre en el aprendizaje del léxico, pueden aparecer errores en las diferentes dimensiones que conforman los emblemas: la forma, el significado, el uso y los exponentes lingüísticos asociados. En la forma, es decir, en la realización kinésica de los emblemas, pueden aparecer errores en las repeticiones, en la velocidad, en las extremidades empleadas, etc., equiparables a los errores fonológicos y gráficos de los elementos verbales. A propósito de lo anterior, Poyatos relaciona la kinésica y la fonología y acuña los términos acento kinésico extranjero (Poyatos, 2017: 22) y acento visual extranjero (Poyatos, 2017: 44). También pueden ocurrir errores en el significado, asociando una forma gestual a un significado incorrecto, y en el uso, empleando un emblema en un contexto inadecuado, por ejemplo, utilizando el emblema /cuernos $/{ }^{9}$ en un contexto formal para expresar que alguien ha sido infiel. Esto sucede igualmente cuando se está aprendiendo una nueva unidad léxica: es posible que se encuentren

2:04); /ガールフレンド/ (/gārufurendo/) (/novia/) https://goo.gl/MuQ857 (Nejokitablog, 2015, minutos 5:55-5:57).

${ }^{9}$ Los dedos índice y meñique de una mano se colocan, con el resto del puño cerrado, verticalmente por encima de la frente. Su realización puede observarse en el siguiente vídeo: https://goo.gl/a1Nhhy (Gordeazábal, 2007, minuto 1:15). 
problemas pragmáticos, discursivos y sociolingüísticos a la hora de usar esas unidades en la comunicación, así como es frecuente que antes de haber logrado su adquisición se asocien formas a significados incorrectos dentro del mismo conjunto de unidades léxicas que se están aprendiendo o dentro de los significados polisémicos u homónimos de una palabra.

Para ilustrar lo anterior relatamos a continuación un ejemplo experimentado recientemente por esta autora en el aula: una estudiante anglohablante de español inicial, ante la pregunta de la profesora ¿Cómo estás?, respondió Estoy vale. Esta estudiante, que había escuchado a su profesora la interjección vale en numerosas ocasiones como equivalente de okay en inglés para expresar asentimiento o conformidad, asumió que los significados y usos léxicos de ese término serían los mismos en español, por lo que para expresar I'm okay, dijo Estoy vale. Es decir, en el proceso de aprendizaje de la interjección, la estudiante todavía no había alcanzado el estadio donde supiera discernir la equivalencia parcial entre el término del inglés okay y el del español de España vale. ${ }^{10}$ No obstante, ese error sirvió (además de para crear un momento divertido en el aula) para que la estudiante (y el resto de la clase) pudieran comprender por qué vale no se podía utilizar en ese contexto. Del mismo modo, esta situación podría ocurrir en el aprendizaje de los emblemas. Por ejemplo, cuando un estudiante de ELE japonés esté aprendiendo cómo expresar 'ok' gestualmente en español, deberá saber:

En primer lugar, que hay dos opciones, de las cuales una es común con su repertorio japonés: (1) formando un círculo con los dedos índice y pulgar mientras que el resto de dedos estirados y separados apuntan hacia arriba con la palma hacia afuera; pero otra no lo es: (2) levantando el dedo pulgar mientras el resto de dedos permanecen en puño cerrado. Esta última forma gestual, de hecho, en japonés pertenece al emblema /ボーイフレンド/ (/bōifurendo/), que significa 'novio' (Hamiru-aqui y Chang, 2004: 94-95). Es decir, este gesto del japonés y /ok 2/ del español son equimorofosdimónimos.

En segundo lugar, que la forma gestual común con significado 'ok' en español y en japonés (/ok 1/), solo se utiliza para expresar 'ok' en español. En japonés, esta forma

\footnotetext{
${ }^{10}$ De hecho, en el uso del término ok/okay en español sucede lo mismo: podría usarse como sinónimo de vale para asentir o expresar conformidad (ej. ¿Quieres salir a comer? / Okay) pero no para expresar bienestar (ej. ¿Cómo estás? / Estoy *okay, en lugar de Estoy bien).
} 
gestual tiene otro significado: según Hamiru-aqui y Chang (2004: 104-105), «if this gesture is made in a drugstore it means a condom». Es decir, en relación con el ejemplo anterior de aprendizaje del léxico, igual que la forma verbal vale solo significa 'ok' como conformidad y asentimiento, la forma gestual de formar un círculo con los dedos índice y pulgar mientras que el resto de dedos estirados y separados apuntan hacia arriba, solamente significa 'ok' en español. En otras palabras, el estudiante japonés deberá aprender que la equivalencia entre el significado de estos emblemas del español y del japonés es parcial y, por ello, en español solo será correcto con el significado de 'ok' y nunca con el de 'condón'.

Los ejemplos presentados, así como las reflexiones teóricas expuestas a lo largo de estas páginas, muestran la similitud en los procesos del aprendizaje del léxico y de los emblemas. Por ello, consideramos apropiado un trabajo conjunto de ambos en la enseñanza de una L2/LE. No obstante, todas las reflexiones anteriores deberán ser verificadas empíricamente con futuras experimentaciones donde se estudien los procesos subyacentes al aprendizaje de estos gestos. Hasta que estas investigaciones puedan llevarse a cabo, es importante contar con una exposición teórico-pedagógica que facilite la incorporación inmediata de estos gestos en el aula de ELE. Seguidamente se exponen una serie de criterios para ello.

\section{Nivelación e integración de los emblemas en el aula de ELE}

\subsection{Criterios de nivelación}

\subsubsection{Graduación}

Igual que sucede en el resto de los elementos de la lengua, los signos no verbales deberían tener una graduación por niveles que pudiera incluirse en los diferentes materiales didácticos. Cestero (2017a: 1110) apunta muy acertadamente que esta graduación deberá realizarse en consonancia con el plan curricular con el que se esté trabajando y teniendo en cuenta criterios como la funcionalidad, la frecuencia de aparición y la dificultad. Otras propuestas de integración de los signos no verbales, y que pueden aplicarse a los emblemas, apuntan que deberán enseñarse de fácil a difícil (Poyatos, 2017), de lo más general a lo más específico, de lo más simple a lo más complejo y de lo más frecuente a lo menos frecuente (Méndez Guerrero, 2016) y se debe comenzar con los signos no verbales que sean semejantes en la lengua materna, lo cual 
servirá, por un lado, para que el estudiante sea consciente de las similitudes entre su lengua y la lengua meta y, por otro lado, para comunicarse no verbalmente ante la falta de recursos verbales en los niveles iniciales (Cabañas Martínez, 2005).

Puesto que no disponemos de ningún estudio de corpus que recoja la frecuencia de aparición de los gestos emblemáticos, ese criterio no podrá servirnos por el momento como pauta para la nivelación. Tampoco contamos con ninguna investigación psicolingüística que mida qué emblemas son más difíciles de aprender en función de las lenguas maternas de los estudiantes, por ello, el grado de dificultad no puede tomarse como pauta niveladora. Sin embargo, en relación con lo que señala Cabañas Martínez (2005), los emblemas (cuasi)equimorfos-sinónimos, esto es, lo que son semejantes en la lengua materna tanto en significante como en significado y uso, serán más fáciles de aprender que el resto. Así, estamos de acuerdo en comenzar con esta categoría emblemática, ya que, además, servirá como actividad de sensibilización hacia estos gestos, es decir, hará que los estudiantes sean conscientes de su propio código emblemático y la funcionalidad del mismo. Ahora bien, esto solo podrá desarrollarse en aulas de ELE donde los alumnos compartan la misma lengua materna. Por ello, en clases lingüísticamente heterogéneas deberá aplicarse una instrucción diferenciada en este primer estadio, lo cual también servirá para después poner en común las reflexiones de cada grupo materno. Esto favorecerá que los estudiantes no solo sean conscientes de su propio código emblemático, sino también de la existencia de diferencias interculturales.

\subsubsection{Recuperación progresiva}

Tanto para los emblemas comunes con la lengua materna como para los que no lo son, en los niveles iniciales deberán asociarse con unidades léxicas más simples que en los subsecuentes intermedios y avanzados. Asimismo, es fundamental subrayar la necesidad de recuperar progresivamente estos gestos a lo largo de los diferentes niveles lingüísticos. Es decir, del mismo modo que sucede con los elementos verbales de la lengua, los emblemas aprendidos en los niveles iniciales deberán ser recordados y ampliados en los intermedios y avanzados. Como para el resto de signos no verbales, este proceso es muy importante para los emblemas, ya que, debido a la falta de atención a la comunicación no verbal en los materiales disponibles, es posible que los estudiantes posean una alta competencia de lenguaje verbal, pero desconozcan por completo el componente emblemático. Por ello, hasta que los signos no verbales se incluyan de manera sistemática en los enfoques curriculares y, por ende, en los programas, 
manuales y materiales, será muy importante que los profesores se adapten a las necesidades de los estudiantes y en función de ellas, decidir cómo y qué gestos emblemáticos son más pertinentes en el aula.

En función del nivel lingüístico, estos gestos se presentarán con unas unidades léxicas u otras, de manera que también puedan aprovecharse para recordar los exponentes lingüísticos conocidos en torno a su núcleo semántico. De esta manera, el gesto /loco/ podrá trabajarse junto con las unidades estar loco o volverse loco en los niveles iniciales e ir progresivamente incorporando otras en los niveles siguientes, como, por ejemplo, estar como una cabra, estar pirado o faltarle un tornillo a alguien. Así, el trabajo con emblemas servirá también para establecer redes paradigmáticas en torno a campos semánticos. En otras palabras, además de las unidades léxicas sinónimas para denominar al gesto emblemático, se podrá seguir trabajando el componente léxico ampliando el vocabulario en torno a agrupaciones conceptuales y funcionales. Esto, además, podrá aprovecharse para trabajar la fraseología del español.

Basándonos en las orientaciones del PCIC (Instituto Cervantes, 2006) y en nuestra experiencia docente, en la Tabla 1 se presenta un ejemplo de secuenciación fraseológica para /loco/. Se clasifica en los tres niveles generales de referencia (A, B y C), pero se señala entre corchetes los casos donde el contenido gramatical y léxico aparece explícitamente en los subniveles del PCIC.

Tabla 1. Secuenciación fraseológica para el emblema /loco/

\begin{tabular}{|c|l|}
\hline Niveles & \multicolumn{1}{|c|}{ Exponentes lingüísticos } \\
\hline A & Estar loco, estar mal, no estar bien. \\
\hline B & $\begin{array}{l}\text { Estar loquísimo [B1], volverse loco [B2], estar como una cabra [B2], estar pirado, } \\
\text { estar mal de la cabeza, no estar bien de la cabeza. }\end{array}$ \\
\hline C & Faltarle un tornillo a alguien, írsele la olla a alguien, estar loco de atar, estar chiflado. \\
\hline
\end{tabular}

Además de la graduación de unidades fraseológicas, también se pueden nivelar los usos pragmáticos y los diferentes significados de los emblemas. Por ejemplo, el gesto /a dos velas/11 del español de España puede significar 'sin dinero' o 'sin relaciones

${ }^{11}$ El puño de una mano se cierra con la excepción de los dedos índice y corazón, que quedan en forma de V. Estos hacen un movimiento rápido (que puede ser repetido) partiendo de los párpados y 
sexuales'12. Así, en los niveles A puede introducirse con el significado 'sin dinero' asociado a la unidad no tener dinero, mientras que en los niveles $\mathrm{B}$ y $\mathrm{C}$ se puede incluir su otro significado, 'sin relaciones sexuales', y recuperarse el significado 'sin dinero' asociándose a otras unidades más complejas como estar a dos velas o quedarse a dos velas, ya que el PCIC introduce por primera vez el término vela en el nivel B2 como medio de transporte para barco de vela y lo recupera, primero, en el nivel C1 solo presentando vela como parte del campo semántico en torno al transporte marítimo y fluvial y, segundo, en el nivel C2 con navegación a vela.

\subsection{Criterios de integración}

\subsubsection{Por unidades temáticas}

En relación con las ideas presentadas anteriormente, para que el aprendizaje de estos gestos sea significativo e integrador, en los cursos de español general lo más adecuado es que se incorporen dentro de unidades temáticas que muestren su funcionalidad y uso en la comunicación. Por ejemplo, /juntos/ y /cuernos/ podrían incorporarse en una unidad dedicada a las relaciones sociales y sentimentales. Así, junto con los exponentes lingüísticos asociados a esos emblemas (estar liados, estar juntos, tener algo, poner los cuernos, ser un cornudo, ser infiel, etc.), se trabajarían otras unidades léxicas que pertenecieran a ese campo semántico, por ejemplo, divorciarse, romper, casarse, salir con alguien, llevarse bien/mal, gustar, tener una cita, etc. Como se ha comentado previamente, las diferentes unidades léxicas deberán escogerse en función del nivel de los estudiantes, de manera que los exponentes lingüísticos asociados a los emblemas, así como los pertenecientes a los campos semánticos donde esos exponentes se incluyen, se vayan ampliando progresivamente. Esto asegurará también la recuperación, práctica y refuerzo del contenido emblemático a lo largo de los diferentes niveles.

terminando en los labios, la nariz queda en medio de los dedos. Su realización puede observarse en el siguiente vídeo: http://bit.ly/2YQBMek (Subirats, 2019, minuto 17:30).

12 En los siguientes vídeos se puede observar su uso con el significado 'sin dinero': http://bit.ly/2YPQe6q (Subirats, 2018b, minuto 18:01) y http://bit.ly/30LFxn4 (Rivera, 2017, minuto 11:51); y con el significado 'sin relaciones sexuales': http://bit.ly/2X7FLTm (Garrido y Montalbán, 2018b, minutos 1:46-1:50). 
Asimismo, este criterio de incorporar los emblemas teniendo en cuenta las líneas temáticas ayudará a integrarlos dentro de los contenidos de los manuales de español, puesto que la mayoría de ellos se organizan en torno a unidades temáticas. Ahora bien, si en algún momento de la unidad aparecieran gestos que no pertenecen al campo semántico del léxico asociado, por ejemplo, en un material real como un vídeo, sería apropiado trabajarlos igualmente, ya que probablemente se despierte el interés de los estudiantes por descubrir su significado para poder entender el contenido del material.

\subsubsection{Por unidades léxicas}

Además del criterio temático, también se podrán trabajar agrupando el tipo de exponentes lingüísticos asociados, por ejemplo, un tipo muy productivo (por la iconicidad de muchos emblemas) son las unidades léxicas que contienen partes del cuerpo: ser un caradura, tener mucha cara o tener más cara que espalda para /caradura/13, ser un cabeza loca para /loco/, estar hasta las narices, hasta el moño o hasta la coronilla para /hasta aquí/14, estar para chuparse los dedos o estar a pedir de boca para /buenísimo/, rugir o crujir las tripas para /hambre/15, (tener) ojo para /ojo/16, ser de puño cerrado/de la cofradía de la virgen del puño para /agarrado/17, ser un codo o doler el codo para /codo/18, etc.

13 Este gesto pertenece a la variedad del español de España y se realiza con el dorso de una mano golpeando rápida y repetidamente la mejilla. Su realización puede observarse en el minuto 0:53 de la película Ahora o Nunca (Ramos y Ripoll, 2015).

14 La palma de una mano hacia abajo, con los dedos estirados, se sitúa a la altura del cuello, la nariz, la frente o encima de la cabeza, pudiéndose mover una o varias ocasiones a velocidad media. Este gesto puede verse en el siguiente vídeo: https://goo.gl/3Dw8yB (Flitz, 2018, minuto 0:00).

15 La palma abierta de una mano toca el estómago haciendo movimientos circulares de velocidad media. Su realización puede observarse en el minuto 18:53 de la película La Jaula de Oro (Alvarado y Quemada-Díez, 2013).

16 La yema del dedo índice de una mano, con el resto de puño cerrado, tira hacia abajo el párpado inferior aumentando la abertura del ojo o golpea repetidamente con una velocidad media debajo del ojo.

${ }^{17}$ Este gesto es propio de España y se realiza levantando el puño cerrado de una mano a la altura del hombro a velocidad media.

${ }^{18}$ Este gesto pertenece a las variedades del español de Bolivia, Colombia, Cuba, Ecuador, Guatemala, Honduras, México, Nicaragua, Panamá, Paraguay, Perú, Salvador, Uruguay y Venezuela, significa 'ser 
Otro tipo de exponentes lingüísticos que pueden aprovecharse para la práctica de emblemas son los que contienen animales o partes de ellos, por ejemplo, estar como una cabra para /loco/, ser un cornudo o poner los cuernos para /cuernos/, ser un loro o una cotorra para /blablablá/ y ser un rata para /agarrado/. Además de los anteriores modismos, donde se produce una asociación directa entre el emblema y el exponente verbal, en los siguientes ejemplos se puede encontrar una relación indirecta: en boca cerrada no entran moscas para / silencio/ ${ }^{19} \mathrm{y} /$ cerrar la boca/20, comer como un pajarillo para /delgado/, tener la mosca detrás de la oreja para /darle vueltas a algo/21, por la boca muere el pez para /blablablá/ y ser astuto como un zorro para /pensar/22, el cual en ocasiones se emplea para señalar que alguien es inteligente. También se puede trabajar con los significados opuestos: ser una foca/una vaca para el emblema /delgado/ y haber cuatro gatos para /mucho/23, que se utiliza frecuentemente para indicar que un lugar está abarrotado de gente.

tacaño' y «se realiza levantando el puño cerrado a la altura del hombro y golpeando el codo de la misma extremidad con la palma de la otra mano» (Meo-Zilio y Mejía, 1980: 43-44).

${ }^{19}$ El dedo índice de una mano de coloca verticalmente sobre los labios. Su realización puede observarse en el minuto 27:14 de la película española Es por tu bien (Barrios, Augustín, Jiménez y Therón, 2017).

${ }^{20}$ Los dedos pulgar e índice de una mano recorren los labios de derecha a izquierda si se utiliza la mano izquierda o de izquierda a derecha si se usa la mano derecha. Se puede encontrar el siguiente vídeo: http://bit.ly/2JIsqOt (Rey y Gálvez, 2019, minuto 1:10).

${ }^{21}$ El dedo índice de una mano, con el resto del puño cerrado, hace movimientos circulares de velocidad rápida, media o lenta apuntando a la sien. Su realización puede verse en el siguiente vídeo: http://bit.ly/2VUeZfZ (Carrasco, 2018, minuto 13:44).

22 El dedo índice de una mano uno, dos o varios golpes rápidos en la sien mientras el resto de dedos permanecen en puño cerrado. En el siguiente vídeo se puede observar la realización con el significado de 'inteligente': http://bit.ly/30PaF58 (Un Catracho en Japón, 2019, minuto 7:36).

${ }^{23}$ Los dedos se apiñan verticalmente con las puntas hacia arriba y se separan en movimientos rápidos y repetidos de apertura y cierre completo hasta que se tocan las yemas del pulgar con las yemas del resto de los dedos. Puede encontrase a continuación: https://goo.gl/grxrhx (Garrido y Montalbán, 2018a, minutos 2:19 y 4:33). 


\subsubsection{Como protagonistas}

Por último, si se trata de un curso específico de cultura, de español oral o de comunicación no verbal, no será necesario organizar los emblemas por campos semánticos ni por tipo de unidades léxicas, sino que estos podrán formar una sección dedicada a la gestualidad del español. Es decir, podrán ser los protagonistas y ejes vertebradores de las actividades, donde, a partir del emblema, se trabajarán las unidades léxicas que el profesor determine para cada gesto en función del nivel de español y del conocimiento previo de los estudiantes.

\subsection{La variación intracultural}

Además de las propuestas anteriores de integración, es importante tratar el asunto de la variación intracultural emblemática. Como se ha podido comprobar a través de algunos ejemplos ofrecidos a lo largo de estas páginas, dentro del mundo panhispánico también existen diferencias emblemáticas, por lo que el estudiante de ELE no solo deberá aprender las diferencias interculturales entre su lengua materna y el español, sino que también deberá, al menos, saber que existe una variedad intrahispánica, como sucede con el componente léxico.

Si bien en las clases de ELE que se den en un país específico parece más productivo que se trabajen principalmente los emblemas de ese lugar, puesto que son los que van a aparecer en la comunicación real con nativos, presentar las diferencias intraculturales en estos contextos también sirve para mostrar la riqueza lingüística del español y preparar a los estudiantes para futuras experiencias interculturales. En el contexto de clases en países extranjeros, presentar y trabajar la variedad intracultural será necesario. Esto debe tenerse en cuenta especialmente para los estudiantes en Estados Unidos, país con una gran población hispana, donde los aprendientes de español pueden comunicarse con nativos de todos los países hispanohablantes.

Por ello, deberán explicarse y trabajarse las diferencias y similitudes emblemáticas teniendo en cuenta las múltiples opciones contrastivas. Así, los estudiantes deberán conocer que dentro del mundo hispanohablante existen, por ejemplo, los equimorfos- 
dimónimos ${ }^{24}$ /mucho/ (de la variedad del español de España) y /miedo/25 (Argentina, Bolivia, Colombia, Costa Rica, Chile, Ecuador, Guatemala, Honduras, México, Nicaragua, Panamá, Perú, Salvador y Uruguay [Meo Zilio y Mejía, 1983: 67-68]); los cuasiequimorfos-sinónimos /sí 1/ y /sí 2/: ambos se realizan con movimientos verticales, pero el no 1 se realiza con la cabeza (en todos los países hispanohablantes) y el no 2 con el dedo índice, el cual se flexiona y se expande (este emblema está presente en la variedad mexicana, pero no en el español de España ${ }^{26}$ ); los dimorfos-sinónimos, mencionados anteriormente, /agarrado/y /codo/; y unimorfos de una sola variedad del español como el ya presentado /a dos velas/ en España. Además, también deberá prestarse atención a los equimorfos-sinónimos, puesto que, aunque las dimensiones de la forma, el significado y el uso coincidan, es posible que haya diferencias en los exponentes lingüísticos: por ejemplo, en el emblema /hasta aquí/ tanto un español como un mexicano dirían estoy hasta aquí, estoy harto o estoy hasta la coronilla, pero en un español podría aparecer estoy hasta la peineta y un mexicano, en cambio, diría estoy hasta la madre 27.

\section{Conclusiones}

La enseñanza de los gestos emblemáticos en las clases de español todavía es un asunto que necesita estudiarse e incorporarse en los procedimientos didácticos de manera sistemática y significativa. A través de una serie de consideraciones teórico-

\footnotetext{
${ }^{24}$ Es importante advertir que en el aula no se debe usar esta terminología lingüística, sino que deberán emplearse circunloquios que sean comprendidos por los estudiantes. Por ejemplo, para los emblemas equimorfos-dimónimos: 'gestos que tienen la misma forma pero que tienen un significado diferente'.

${ }^{25}$ Ambos se realizan con la mano vertical y dedos estirados realizando varios movimientos de apertura y cierre completo hasta que se tocan las yemas del pulgar con las yemas del resto de los dedos. /Miedo/ puede observarse en la película peruana Lusers (Alcántara y Rodríguez, 2015, minuto 1:24:00). Para /mucho/, véase la nota no 23.

${ }^{26}$ Resulta interesante que el repertorio emblemático de España, a pesar de que no contenga /sí 2/, sí se encuentran /no 1/ y /no 2/: en ambos se realizan movimientos laterales, pero en uno se emplea el dedo índice y en otro la cabeza.

${ }^{27}$ En el siguiente vídeo se puede observar a un hablante mexicano realizando este emblema junto con el exponente lingüístico estoy hasta la madre: http://bit.ly/2Ey6tgP (Micha, 2017, minuto 0:12)
} 
pedagógicas acerca de la relación entre el componente emblemático y léxico de la lengua, se ha expuesto la similitud en los procesos cognitivos que se producen cuando se aprende una nueva unidad léxica y un nuevo emblema: como parte de la red mental donde se organizan las categorías que permiten denominar el mundo, en el lexicón se almacenan, junto con las palabras y unidades léxicas, las formas gestuales.

Lo anterior deberá ser comprobado empíricamente a través de investigaciones que estudien el aprendizaje de estos gestos y sustenten las prácticas didácticas. Hasta que estos estudios puedan llevarse a cabo, en este trabajo se han ofrecido una serie de criterios que faciliten la incorporación inmediata de los emblemas en el aula de ELE. Estos gestos deberán integrarse junto con el resto de los elementos lingüísticoculturales, estableciendo redes paradigmáticas en torno a los campos semánticos de los exponentes lingüísticos asociados. Como parte de los objetivos curriculares, los emblemas y sus unidades léxicas deberán graduarse y recuperarse progresivamente en los diferentes niveles del español.

Por último, además de la competencia léxica, el trabajo emblemático deberá incluirse junto con el desarrollo de la conciencia y el dominio, por un lado, intercultural, donde los estudiantes deberán aprender las diferencias entre su lengua materna y el español y, por otro lado, intracultural, donde deberán conocer la variación emblemática dentro del mundo panhispánico.

\section{Referencias bibliográficas}

\section{Obras citadas}

ACTFL (2012). ACTFL Performance Descriptors for Language Learners. Alexandria, VA: The American Council on the Teaching of Foreign Languages.

ACTFL (2015). ACTFL Performance Descriptors for Language Learners. Alexandria, VA: The American Council on the Teaching of Foreign Languages.

BARALO, M. (1997). «La organización del lexicón en la lengua extranjera». Revista de Filología Románica, 1 (14): 59-71.

BARALO, M. (2001). «La construcción del lexicón en español / LE: transferencia y construcción creativa». En: M. A. MARTÍN ZORRAQUINO y C. DÍEZ PELEGRÍN (eds.). Actas del XI Congreso Internacional de ASELE. ¿Qué español enseñar?: Norma y variación lingüísticas en la enseñanza del español a extranjeros, Zaragoza. ASELE/Universidad de Zaragoza: 165-174. 
BARBIERI, F., A. BUONOCORE, R. DALLA VOLTA y M. GENTILUCCI (2009). «How symbolic gestures and words interact with each other». Brain \& Language, 110: 111.

BELÍO APAOLAZA, H. S. (2018). Aprendizaje y evaluación de la comunicación no verbal en ELE. Propuesta teórica y estudio empírico sobre los gestos emblemáticos. [Tesis doctoral]. Salamanca: Universidad de Salamanca.

CABAÑAS MARTÍNEZ, M. J. (2005). «Comunicación no verbal y enseñanza de español como segunda lengua a inmigrantes en contextos escolares». Interlingüística, 16: 225-236.

CESTERO, A. M. (2017a). «La comunicación no verbal». En: A. M. CESTERO MANCERA y I. PENADÉS MARTÍNEZ (eds.). Manual del profesor de ELE. Alcalá de Henares. Universidad de Alcalá: 1051-1122.

CESTERO, A. M. (2017b). «Comunicación no verbal y diversidad cultural: necesidades y recursos en EL2 y ELE». En: D. G. NÍKLEVA (ed.). Necesidades y tendencias en la formación del profesorado de español como lengua extranjera. Bern. Peter Lang: 339-382.

CONSEJO DE EUROPA E INSTITUTO CERVANTES (Trad.) (2002). Marco común europeo de referencia para las lenguas: aprendizaje, enseñanza, evaluación. Madrid: MECDAnaya.

CONSEJO DE EUROPA (2018). Common European Framework of references for languages: learning, teaching, assessment. Companion volume with new descriptors. Sin Ciudad: Consejo de Europa.

DAHAN, G. y J. COSNIER (1977). «Sémiologie des quasi-linguistiques français». Psychologie Médicale, 9 (11): 2053-2072.

DURÃO, A. B. A. B. (2007). La interlengua. Madrid: Arco Libros.

GENTILUCCI, M., R. DALLA VOLTA y C. GIANELLI (2008). «When the hands speak». Journal of Physiology, 102: 21-30.

GÓMEZ MOLINA, J. R. (2004). «La subcompetencia léxico-semántica». En: J. SÁNCHEZ LOBATO y I. SANTOS GARGALLO (dirs.). Vademecum para la formación de profesores: enseñar español como segunda lengua (L2)/lengua extranjera(LE). Alcobendas. SGEL: 491-510.

HAMIRU-AQUI y A. CHANG (2004). 70 Japanese Gestures. No Language Communication. Tokio: IBC Publishing.

INSTITUTO CERVANTES (2006). Plan curricular del Instituto Cervantes. Niveles de referencia para el español. Madrid: Instituto Cervantes-Biblioteca nueva.

KENDON, A. (1988). «How gestures can become like words». En: F. POYATOS (ed.). Cross-cultural perspectives in nonverbal communication. Nueva York. Hogrefe: 131141.

MARCONI, D. (1997). Lexical competence. Cambridge, MA: The MIT Press.

MATSUMOTO, D. y H.C. HWANG (2013). «Cultural Similarities and Differences in Emblematic Gestures». Journal of Nonverbal Behaviour, 37 (1): 1-27.

MÉNDEZ GUERRERO, B. (2016). «El componente no verbal en el aula de ELE. Consideraciones para su aprendizaje significativo e intercultural a partir de 
WebQuest». Revista Nebrija de Lingüística Aplicada a la Enseñanza de las Lenguas, 20: 1-14.

MEO-ZILIO, G. y S. MEJÍA (1980). Diccionario de gestos: España e Hispanoamérica, Vol I. Bogotá: Insituto Caro Cuervo.

MEO-ZILIO, G. y S. MEJÍA (1983). Diccionario de gestos: España e Hispanoamérica, Vol II. Bogotá: Insituto Caro Cuervo.

NCSSFL-ACTFL (2014). Intercultural can-do statements. Alexandria, VA: The National Council of State Supervisors for Languages y The American Council on the Teaching of Foreign Languages.

NCSSFL-ACTFL (2017). NCSSFL-ACTFL can do statements. Alexandria, VA: The National Council of State Supervisors for Languages y The American Council on the Teaching of Foreign Languages.

PAYRATÒ, L. (2003). «What does 'the same gesture' mean? A reflection on emblems, their organization and their interpretation». En: M. RECTOR, I. POGGI y N. TRIGO (eds.). Gestures, Meaning and Use. Porto. Fernando Pessoa University Press: 73-81.

POGGI, I. y M. ZOMPARELLI (1987). «Lessico e grammatica nei gesti e nelle parole». En: I. POGGI (ed.). Le parole nella testa: Guida a un'educazione linguistica cognitivista. Bolonia. Il Mulino: 291- 327.

POYATOS, F. (1994). La comunicación no verbal I. Cultura, lenguaje y conversación, Madrid: Istmo.

POYATOS, F. (2017). «La comunicación no verbal en la enseñanza integral del Español como Lengua Extranjera». En: A. M. Cestero (dir.). E-eleando, ELE en la Red. Serie de monografías y materiales para la enseñanza de ELE. Alcalá de Henares: Universidad de Alcalá Servicio de Publicaciones.

WILLEMS, R. M., A. ÓZYÜREK y P. HAGOORT (2007). «When language meets action: The neural integration of gesture and speech». Cerebral Cortex, 17: 2322-2333.

XU, J., P. J. GANNON, K. EMMOREY, J. F. SMITH y A. R. BRAUN (2009). «Symbolic gestures and spoken language are processed by a common neural system». Proceedings of the National Academy of Sciences of the United States of America, 109 (49): 2066420669.

\section{Recursos audiovisuales}

ALCÁNTARA, C. (prod.) y T. RODRÍGUEZ (dir.) (2015). Lusers [cinta cinematográfica]. Perú y Chile: Tondero Films y Bamboosa.

ALVARADO, D. (prod.) y D. QUEMADA-DÍEZ (dir.) (2013). La Jaula de Oro [cinta cinematográfica]. México: Animal de Luz Films, Machete Producciones.

BARRIOS, G., A. Augustín, E. Jiménez (prods.) y C. Therón (dir.) (2017). Es por tu bien [cinta cinematográfica]. España: Telecinco Cinema y Quexito Films.

CARRASCO, G. (prod. ejec.) (17/06/2018). «Chester: My Way» [archivo de vídeo]. En Chester [programa de televisión]. España: La Fábrica de la Tele y Mediaset España. Disponible en: http://bit.ly/2VUeZfZ, consultado el 24 de mayo de 2018. 
CHIVER, M., M. VARGAS (prods.) y G. ALAZRAKI (dir.) (2019). «Todos somos Cuervos», episodio 12, temporada 4 [capítulo de serie]. En M. Chiver y Vargas, M., Club de Cuervos. México: Netflix y Alazraki Entertainment.

EL VICHO (10/07/2018). «Gestos japoneses - Japonés 101» [archivo de vídeo]. Disponible en: http://bit.ly/2Ziz7uE, consultado el 27 julio de 2019.

FLITZ, M. (2018). «¡No voy a la huelga de mujeros!» [archivo de vídeo]. En El HuffPost. Disponible en: https://goo.gl/3Dw8yB, consultado el 6 de octubre de 2018.

GARRIDO, T. y C. MONTALBÁN (prods.) (01/03/2018a). «Chica Fitness contra los haters» [archivo de vídeo]. En Vodafone Yu [programa de radio]. España: Los 40 Principales, Vodafone y Wink TTD. Disponible en: https://goo.gl/grxrhx, consultado el 6 de octubre de 2018.

GARRIDO, T. y C. MONTALBÁN (prods.) (27/09/2018b). «Victoria Martín (Chica Fitness) le declara su amor a Miguel Ángel Muñoz» [archivo de vídeo]. En Vodafone $Y u$ [programa de radio]. España: Los 40 Principales, Vodafone y Wink TTD. Disponible en: http://bit.ly/2X7FLTm, consultado el 27 de mayo de 2019.

GARRIDO, T. y C. MONTALBÁN (prods.) (02/10/2018c). «Paula Echevarría, Maribel Verdú y Juana Acosta nos enseñan su whatsapp» [archivo de vídeo]. En Vodafone $Y u$ [programa de radio]. España: Los 40 Principales, Vodafone y Wink TTD. Disponible en: http://bit.ly/20pSQI1, consultado el 29 de Julio de 2019.

GORDEAZABAL, J. (prod. ejec.) (2007). Vaya semanita: Hablar por gestos [programa de televisión]. San Sebastián: Pausokoa, EITB. Disponible en: https://goo.gl/MDF3G5, consultado el 6 de octubre de 2018.

MICHA, A. (24/08/2017). «Aleks Syntek odia el reggaetón» [archivo de vídeo]. En La Saga [programa de televisión]. Disponible en: http://bit.ly/2Ey6tgP, consultado el 6 de noviembre de 2018.

NEJOKITABLOG (25/10/2015). «Adivinando gestos japoneses (Español vs japonesa)» [archivo de vídeo]. Disponible en: https://goo.gl/MuQ857, consultado el 6 de octubre de 2018.

NEJOKITABLOG (23/06/2016). «Japonesa adivinando gestos españoles» [archivo de vídeo]. Disponible en: http://bit.ly/2VSbNBe, consultado el 6 de octubre de 2018.

RAMOS, P. (prod. ejec.) y M. RIPOLL (dir.) (2015). Ahora o nunca [cinta cinematográfica]. España: A. I. E. Atresmedia Cine; AXN; Canal+ España; Telefónica Studios.

REY, M. y B. GÁLVEZ (prods. ejec.) (13/04/2019). «Anabel Alonso rompe a llorar en la cata final» [archivo de vídeo]. En Máster Chef Celebrity España, temporada 2 [programa de televisión]. España: Gestmusic (Endemol Shine Iberia) y Corporación Radio y Televisión Española S.A. Disponible en: http://bit.ly/2JIsqOt, consultado el 27 de mayo de 2019.

RIVERA, Y. (prod. ejec.) (17/04/2017). «Mi Cámara y Yo: El Rastro de Madrid» [Archivo de vídeo]. En Mi Cámara y Yo [programa de televisión]. España: El Torreón de Sol y Telemadrid. Disponible en: http://bit.ly/30LFxn4, consultado el 27 de mayo de 2019.

RTVE (2018). «Paquita Salas entrega el Goya a Actriz Revelación» [archivo de vídeo]. Disponible en: https://goo.gl/6wAbjy, consultado el 6 de octubre de 2018. 
SUBIRATS, X. (prod. ejec.) (11/06/2018a). «La Resistencia: Entrevista a Beatriz Rico» [archivo de vídeo]. En La Resistencia [programa de televisión]. España: El Terrat y Movistar+. Disponible en: http://bit.ly/2X8hInx, consultado el 27 de mayo de 2019.

SUBIRATS, X. (prod. ejec.) (08/02/2018b). «La Resistencia: Brisa Fenoy y el reggaetón feminista» [archivo de vídeo]. En La Resistencia [programa de televisión]. España: El Terrat y Movistar+. Disponible en: http://bit.ly/2ECIgG0, consultado el 27 de mayo de 2019.

SUBIRATS, X. (prod. ejec.) (23/01/2019). «La Resistencia: Entrevista a Laia Sanz» [archivo de vídeo]. En La Resistencia [programa de televisión]. España: El Terrat y Movistar+. Disponible en: http://bit.ly/2YQBMek, consultado el 27 de mayo de 2019.

UN CATRACHO EN JAPÓN (23/03/2019). «Palabras hondureñas vs salvadoreñas» [archivo de vídeo]. Disponible en: http://bit.ly/30PaF58, consultado el 27 de mayo de 2019.

VELO PUIG-DURÁN, L. y G. GÓMEZ SANCHA (prods. ejec.) (25/12/2017). «Odio la Navidad» [archivo de vídeo]. En Sin Filtros [programa de televisión]. España: Cuerdos de Atar Producciones S.L. Disponible en: http://bit.ly/2MbU6gB, consultado el 27 de mayo de 2019. 